\title{
Successful Use of Early Percutaneous Dilatational Tracheotomy and the No Sedation Concept in Respiratory Failure in Critically Ill Obese Subjects
}

\author{
Sven Kaese MD, Marie Christine Zander MD, and Pia Lebiedz MD
}

\begin{abstract}
BACKGROUND: The prevalence of obesity in developed countries is rising. Currently, Europe has a prevalence of 9-30\% with significant impact on public health systems. Obese patients in ICUs require special management and treatment. Altered anatomy in obese patients complicates procedures such as mechanical ventilation. Obesity affects cardiopulmonary physiology and requires elevated ventilation pressures. In our retrospective study, we determined the effect of early percutaneous dilatational tracheotomy (PDT) and cessation of sedation on respiratory parameters in severely obese subjects. METHODS: From June 2010 to July 2014, we included all subjects with a body weight of $>130 \mathrm{~kg}$ (body mass index $>35 \mathrm{~kg} / \mathrm{m}^{2}$ ) and respiratory failure who were admitted to the medical ICU of the University Hospital of Münster. All subjects were treated with early PDT and immediate cessation of sedative drugs. We compared ventilator parameters and blood gas analysis before and after PDT. Parameters were recorded on days $0,1,3$, and 5. Day 0 represents values during ventilation via an endotracheal tube, and days $1,3,5$ represent values during ventilation via a tracheotomy tube. PDT was performed on day 0 after recording values during ventilation via an endotracheal tube. RESULTS: We included 23 subjects with a mean body mass index of $53.1 \mathrm{~kg} / \mathrm{m}^{2}$ and respiratory failure. After PDT and cessation of sedation, the required ventilation pressures and $\mathrm{F}_{\mathrm{IO}_{2}}$ could be rapidly reduced $(P<.001)$, whereas blood gas parameters significantly improved. We observed no severe PDT-associated complications in our cohort. CONCLUSIONS: In severe obesity, respiratory failure might be increased by problems in mechanical ventilation due to required high pressures and obesity-induced pulmonary restriction. Rapid tracheotomy with reduction of dead-space ventilation and airway resistance as well as cessation of sedation to enable spontaneous breathing might be a key factor in the therapy of respiratory failure. Key words: respiratory failure; percutaneous dilatational tracheotomy; obesity; mechanical ventilation; sedation; intensive care unit. [Respir Care 2016;61(5):615-620. (C) 2016 Daedalus Enterprises]
\end{abstract}

\section{Introduction}

In European countries, obesity prevalence is $9-30 \%$ and rises continuously, ${ }^{1}$ with significant impact on public

Dr Kaese is affiliated with the Division of Electrophysiology, Department of Cardiovascular Medicine, University of Münster, Münster, Germany. Ms Zander and Dr Lebiedz are affiliated with the Division of Cardiology, Department of Cardiovascular Medicine, University of Münster, Münster, Germany.

The authors have disclosed no conflicts of interest.

Dr Kaese presented a version of this paper at the 46th Gemeinsame Jahrestagung der Deutschen Gesellschaft für Internistische Intensivmedizin und Notfallmedizin (DGIIN) und der Österreichischen Gesellschaft für Internistische und Allgemeine Intensivmedizin und Notfallmedizin, held June 2014, in Salzburg, Austria and at the 44th Critical Care Con- health systems. Obese patients are a steadily increasing group of patients in ICUs who require specifically adapted management, treatment, and care. A study in obese subjects showed higher rates of ICU admission, tracheotomy, and mechanical ventilation. ${ }^{2}$

gress of the Society of Critical Care Medicine, held January 2015, in Phoenix, Arizona. Dr Lebiedz presented a version of this paper at the 35th International Symposium on Intensive Care and Emergency Medicine (ISICEM), held March 2015, in Brussels, Belgium.

Correspondence: Sven Kaese MD, Division of Electrophysiology, Department of Cardiovascular Medicine, University of Münster, AlbertSchweitzer-Campus 1, A1, 48149 Münster, Germany. E-mail: sven.kaese@ukmuenster.de.

DOI: $10.4187 /$ respcare. 04333 
Obesity causes serious alterations of cardiopulmonary physiology that is already restricting physical fitness and quality of life without underlying disease and can exacerbate the clinical cause of additionally occurring critical illness. For example, obese patients suffering from influenza A (H1N1) are at higher risk of admission to ICU and death. ${ }^{3}$ Obese patients often suffer from obstructive sleep apnea, obesity hypoventilation, ARDS, hypertension, ischemic heart disease, hyperlipidemia, and type 2 diabetes mellitus. ${ }^{1,4,5}$ Respiratory physiology in obese patients is characterized by reduction of tidal volume, expiratory reserve volume, residual volume, total lung capacity, functional residual capacity, vital capacity, $\mathrm{FEV}_{1}, \mathrm{FVC}$, lung compliance, chest wall compliance, maximum voluntary ventilation, maximum oxygen consumption, diffusing lung capacity for carbon monoxide, upper and lower airway resistance, and increased pleural pressure and work of breathing with unchanged $\mathrm{FEV}_{1} / \mathrm{FVC}^{1,4-6}$ Function of the diaphragm is hampered in obesity due to increased intraabdominal pressure, which additionally contributes to limited compliance of the respiratory system and decreased static and dynamic lung volumes. ${ }^{1,4-6}$ Further, pleural pressure is increased in obese patients, resulting in reduced transpulmonary pressure, and thereby enables collapse of airways and lung parenchyma and generation of atelectasis. ${ }^{1}$ Atelectasis diminishes lung volume and subsequently the area for gas exchange. ${ }^{1}$ However, prone position performed in ARDS patients to reduce atelectasis and to improve arterial oxygenation can be applied in obese patients safely. ${ }^{7}$

Ciaglia et $\mathrm{a}^{8}$ originally described the technique of percutaneous dilatational tracheotomy (PDT) in 1985, which has developed into the standard procedure for ICU patients requiring long-term mechanical ventilation. ${ }^{9}$ Obesity was initially deemed to be a relative contraindication for PDT, but several studies showed that this procedure could be safely performed in obese subjects. ${ }^{9-12}$

A study by Pelosi et al ${ }^{13}$ in sedated, paralyzed, morbidly obese subjects undergoing general anesthesia for abdominal surgery found during the postoperative period a reduction of compliance and increase of resistance of the respiratory system, reduced functional residual capacity, increased work to inflate lungs and chest wall, and reduced pulmonary oxygenation index. Extremely obese patients have longer durations of mechanical ventilation and prolonged stays in the ICU compared with normal-weight patients. ${ }^{14,15}$ Higher ventilation pressures are needed to enable sufficient tidal volumes compared with normalweight patients. Therefore, we conducted this retrospective study in obese subjects with early percutaneous dilatational tracheotomy to investigate the effect of rapid termination of sedation and reduced dead-space ventilation on respiratory and blood gas parameters.

\section{QUICK LOOK}

\section{Current knowledge}

The prevalence of obesity is rising, and obese patients represent a growing group in ICUs who require special management and treatment. Altered anatomy in obese patients complicates procedures such as percutaneous dilatational tracheotomy (PDT) and mechanical ventilation. Obesity affects cardiopulmonary physiology, leading to obesity-induced pulmonary restriction and elevated ventilation pressures. Further, general anesthesia has a more profoundly negative impact on pulmonary physiology in obese patients than in non-obese patients.

\section{What this paper contributes to our knowledge}

PDT could be safely applied in our group of severely obese subjects. Early PDT and cessation of sedation enabled spontaneous breathing, which contributed to reduction of ventilation pressures and $\mathrm{F}_{\mathrm{IO}_{2}}$ in severely obese subjects. Improved ventilator parameters enabled lung-protective ventilation strategies. Further, the treatment strategy of early PDT and cessation of sedation led to improvement of blood gas parameters in severely obese subjects.

\section{Methods}

We performed this retrospective study according to the principles of the 1964 Declaration of Helsinki. Due to the retrospective nature of the study, the IRB approved the study, but waived the requirement for consent and full review.

\section{Study Population and Parameters}

From June 2010 to July 2014, we included all consecutive patients admitted to the intensive care unit for internal medicine of the University Hospital of Münster with a body weight of $>130 \mathrm{~kg}$ (body mass index $>35$ $\mathrm{kg} / \mathrm{m}^{2}$ ) and respiratory failure. Respiratory failure was defined using the Berlin definition of ARDS. ${ }^{16}$ Early PDT was a standard of care in this particular group of morbidly obese subjects in our ICU. We compared ventilator parameters, blood gas analysis, and required oxygen supplementation before and after PDT. Data were analyzed at days $0,1,3$, and 5 . The values at day 0 were collected when subjects were ventilated via an endotracheal tube. Thereafter, PDT was performed at day 0 . Data at days 1, 3, and 5 represent parameters during placement of a tracheotomy tube.

We registered the following parameters: age, sex, body height, body weight, body mass index, duration of venti- 

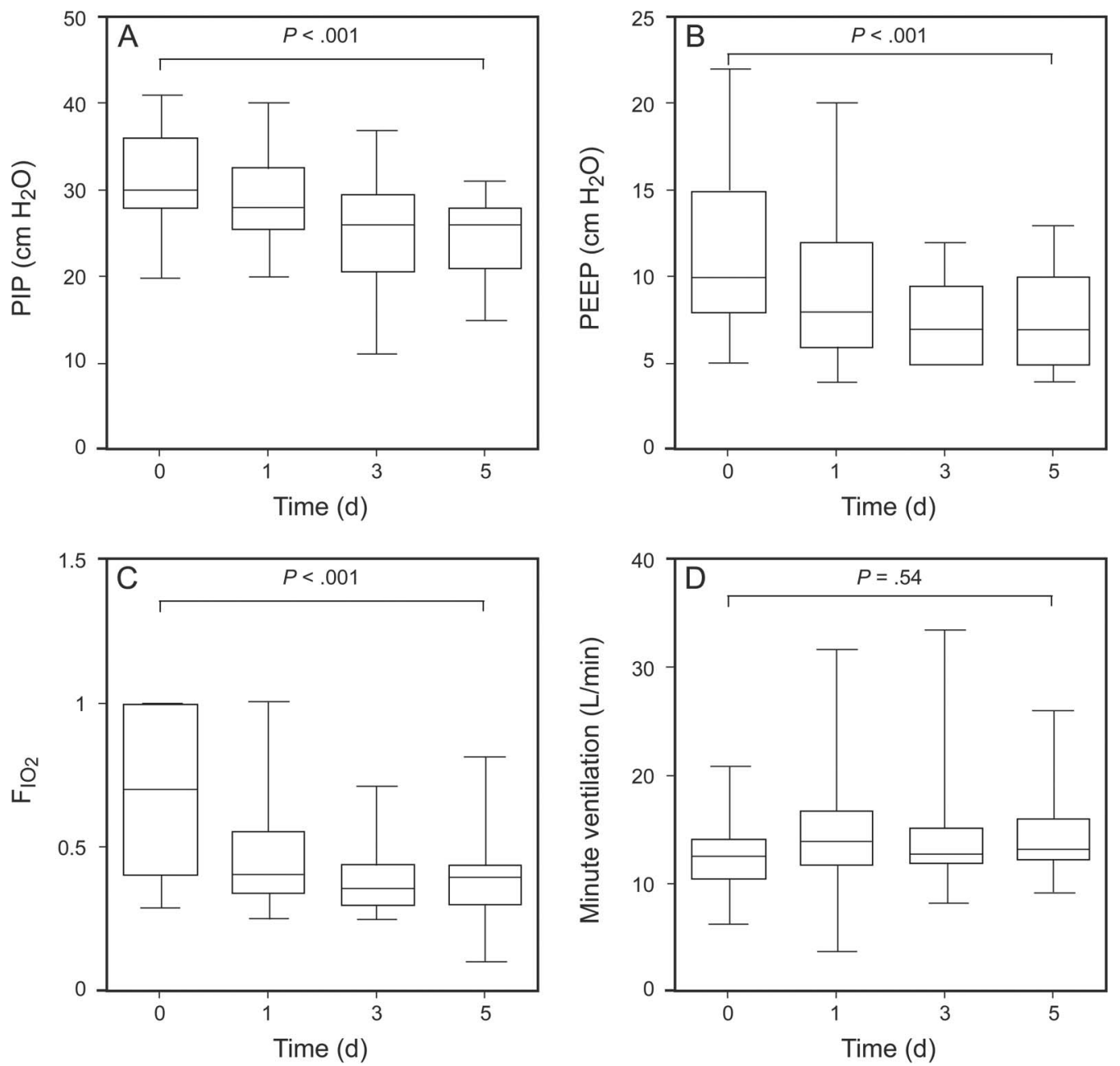

Fig. 1. A: Day 0, $n=23$; day 1, $n=22$; day 3, $n=16$; day 5, $n=13$. B: Day $0, n=23$; day $1, n=23$; day 3, $n=21$; day 5, $n=18$. C: Day $0, n=23$; day $1, n=23$; day $3, n=22$; day $5, n=18$. D: Day $0, n=22$; day $1, n=22$; day $3, n=18$; day $5, n=13$. The decreasing numbers of subjects and parameters are due to successful weaning from mechanical ventilation and enabling spontaneous breathing via tracheotomy tube, successful removal of the tracheotomy tube, and, to a lesser extent, mortality. The center lines in boxes represent the median. Boxes indicate percentiles 25-75, and whiskers represent percentiles 2.5-97.5. The values at day 0 were collected when subjects were ventilated via an endotracheal tube. Thereafter, percutaneous dilatational tracheotomy was performed at day 0 . Data at days 1,3 , and 5 represent parameters during placement of a tracheotomy tube.

lation via endotracheal tube before tracheotomy, duration of ventilation via tracheotomy, complications during tracheotomy, admission diagnosis, preexisting cardiac and pulmonic diseases, Simplified Acute Physiology Score II, and Sequential Organ Failure Assessment score. Investigated ventilator parameters included peak inspiratory pressure, PEEP, expiratory minute volume, and $\mathrm{F}_{\mathrm{IO}_{2}}$. Studied blood gas parameters included arterial partial pressure of $\mathrm{CO}_{2}\left(\mathrm{P}_{\mathrm{aCO}_{2}}\right)$ and of $\mathrm{O}_{2}\left(\mathrm{P}_{\mathrm{aO}_{2}}\right), \mathrm{pH}, \mathrm{HCO}_{3}$, hemoglobin, base excess, and lactate.

Usage of analgo-sedative medication was also recorded. Further, periprocedural complications of PDT like bleeding with a need for treatment and pneumothorax were evaluated.

\section{Technique of PDT}

We performed the modified PDT with the Ciaglia Blue Rhino system (Cook Medical, Bloomington, Indiana). All tracheotomies were performed using the Ultraperc kit (Smiths Medical International Ltd, Hythe, United Kingdom).

\section{Statistical Analysis}

All statistical analyses were performed using PASW 20 (IBM Corp, Armonk, New York). All values in Figures 1 and 2 are displayed as a box and whisker plot (median, tops and bottoms of boxes represent 25th and 75th percentiles and whiskers represent percentiles 2.5 

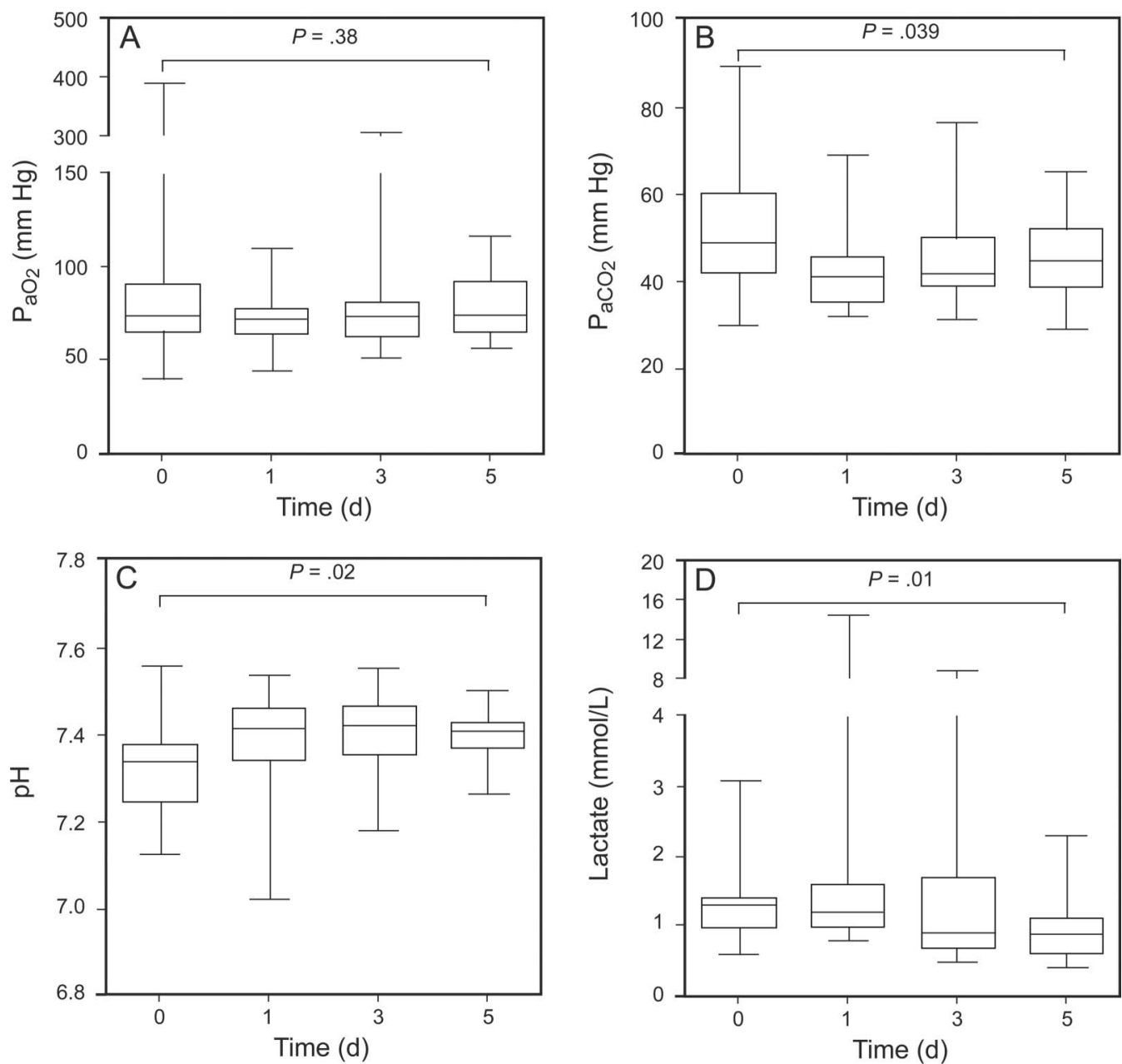

Fig. 2. A: Day 0, $n=23$; day $1, n=23$; day 3, $n=23$; day 5, $n=21$. B: Day $0, n=23$; day $1, n=23$; day 3, $n=23$; day $5, n=21$. C: Day $0, n=23$; day $1, n=23$; day $3, n=23$; day $5, n=21$. D: Day $0, n=23$; day $1, n=23$; day $3, n=23$; day $5, n=21$. The center lines in boxes represent the median. Boxes indicate percentiles $25-75$, and whiskers represent percentiles 2.5-97.5. The values at day 0 were collected when subjects were ventilated via an endotracheal tube. Thereafter, percutaneous dilatational tracheotomy was performed at day 0 . Data at days 1,3 , and 5 represent parameters during placement of a tracheotomy tube.

and 97.5). Values in Table 1 are mean \pm SD. Associations between binary parameters were assessed using the chi-square test. Parameters before and after tracheotomy were compared using the Friedman test. Statistical significance was assumed by a $P$ value of $<.05$, $P$ values of $<.01$ were regarded as highly significant.

\section{Results}

We included 23 severely obese subjects (65.2\% male) with a mean age of $54.4 \pm 11.9$ y who were admitted to our medical ICU. The body mass index was $53.1 \pm 12$ $\mathrm{kg} / \mathrm{m}^{2}$. The most frequent admission diagnosis was respiratory failure due to pneumonia. Most of the subjects $(82.6 \%)$ were transferred from other hospitals to our department due to respiratory failure. Based on the Berlin definition of ARDS, ${ }^{16} 6$ subjects had mild, 9 subjects had moderate, and 8 subjects had severe respiratory failure. We performed an early PDT after an average time of $2.4 \pm 1.4 \mathrm{~d}$ of ventilation via endotracheal tube. Directly after tracheotomy, sedative drugs were ceased. Duration of respiratory support via tracheotomy was $9.8 \pm 6.8 \mathrm{~d}$. During the study period, 2 subjects $(8.7 \%)$ died due to cerebral edema and multi-organ dysfunction (Table 1). In 2 subjects (8.7\%), the tracheotomy tube could successfully be removed at days 3 and 4 . In our study population, we did not observe tolerance problems of the tracheotomy tube or mechanical ventilation.

\section{Respiratory Parameters After PDT}

In our cohort of morbidly obese subjects, the highest level of peak inspiratory pressure, PEEP, and $\mathrm{F}_{\mathrm{IO}_{2}}$ were required when subjects were ventilated via an endotra- 
Table 1. Subjects' Characteristics

\begin{tabular}{lc}
\hline \multicolumn{1}{c}{ Characteristics } & Values \\
\hline$N$ & 23 \\
Male sex, $n(\%)$ & $15(65.2)$ \\
Age, mean \pm SD y & $54.4 \pm 11.9$ \\
BMI, mean \pm SD kg/m ${ }^{2}$ & $53.1 \pm 12$ \\
Transfer from other hospital, $n(\%)$ & $19(82.6 \%)$ \\
Admission diagnosis, $n(\%)$ & \\
$\quad$ CPR & $4(17.4)$ \\
$\quad$ Sepsis & $4(17.4)$ \\
Pneumonia & $11(47.8)$ \\
$\quad$ Heart failure & $4(17.4)$ \\
SAPS II, mean \pm SD & $70.7 \pm 20.5$ \\
SOFA score, mean \pm SD & $14 \pm 4$ \\
Duration of respiratory support, mean \pm SD d & \\
$\quad$ Via endotracheal tube & $2.4 \pm 1.4$ \\
$\quad$ Via tracheotomy & $9.8 \pm 6.8$ \\
Mortality, $n(\%)$ & $2(8.7)$ \\
& \\
\hline CPR $=$ cardiopulmonary resuscitation & \\
BMI $=$ body mass index & \\
SAPS $=$ Simplified Acute Physiology Score II & \\
SOFA $=$ Sequential Organ Failure Assessment & \\
\hline
\end{tabular}

cheal tube at day 0 before we performed PDT. Within the first $72 \mathrm{~h}$ after PDT, required peak inspiratory pressure $(P<.001)$ as well as PEEP $(P<.001)$ and $\mathrm{F}_{\mathrm{IO}_{2}}(P<.001)$ could quickly be reduced (Fig. 1, A-C). Despite significant reduction of ventilator pressures, minute volume was kept constant (Fig. 1D).

\section{Trend of Arterial Blood Gas Analysis}

Despite rapid reduction of PEEP and $\mathrm{F}_{\mathrm{IO}_{2}}$ (Fig. 1), $\mathrm{P}_{\mathrm{aO}_{2}}$ remained constant (Fig. 2A). $\mathrm{P}_{\mathrm{aCO}_{2}}, \mathrm{pH}$, and serum lactate significantly improved after PDT (Fig. 2, B-D).

\section{Complication Rate of PDT}

In our cohort, we did not observe any complications due to the percutaneous tracheotomy procedure. We saw neither cannula dislocation, bleeding complications, nor pneumothorax.

\section{Discussion}

We herein investigated for the first time the effect of early PDT and cessation of sedation after tracheotomy on ventilator and blood gas parameters of morbidly obese subjects. Our data suggest a rapidly occurring positive influence on these parameters by early PDT and early wake up or no sedation strategy in critically ill obese subjects. To enable an early wake up as well as a secure airway management and lung-protective ventilation strat- egy, we prefer an early PDT, which could be safely performed in our group of subjects.

Previous studies have shown that body mass index is positively associated with longer ICU stay and longer duration of mechanical ventilation. ${ }^{17}$ Even an increased mortality attributed to high body mass index has been postulated in patients with acute lung injury. ${ }^{18}$ This is most probably due to a pulmonary restrictive syndrome caused by morbid obesity combined with reduced functional residual capacity. General anesthesia further decreases functional residual capacity and consequently alters gas exchange more profoundly in morbidly obese patients than in non-obese patients. ${ }^{19}$

Given this evidence, mechanical ventilation of morbidly obese patients without sedation by using early tracheotomy seems preferable compared with an endotracheal tube for several reasons: (1) PDT with subsequent cessation of general anesthesia enables spontaneous breathing and leads to a reduction of lung restriction, (2) PDT reduces airway resistance by usage of a tracheal tube with a larger inside diameter, and (3) tracheotomy reduces dead-space ventilation due to a shorter artificial airway.

As described previously, ${ }^{10}$ we could not detect an elevated complication rate assigned to the procedure of percutaneous tracheotomy in our cohort of obese subjects. Because we did not detect severe complications, such as cannula dislocation, bleeding, or pneumothorax due to PDT, we believe that early PDT probably does not contribute to mortality in this group of subjects.

However, several limitations of our study have to be addressed. First, there was a low number of subjects as a result of the single center design. Second, we conducted a retrospective analysis. To prove the benefit of early tracheotomy in morbidly obese subjects, a prospective randomized trial has to be conducted. Furthermore, the no sedation concept has to be compared for subjects with early tracheotomy and subjects with endotracheal intubation to differentiate between effects of reduction of deadspace ventilation and airway resistance by tracheotomy and those effects achieved by wake up.

\section{Conclusions}

The early PDT procedure and a rapid termination of sedation seem to improve ventilator and blood gas parameters. Improving ventilator parameters by reducing peak inspiratory pressure and PEEP may contribute to lungprotective ventilation strategies in morbidly obese patients.

\section{REFERENCES}

1. Hibbert K, Rice M, Malhotra A. Obesity and ARDS. Chest 2012; 142(3):785-790. 


\section{Early PDT and Sedation Cessation in Obese ICU Subjects}

2. Westerly BD, Dabbagh O. Morbidity and mortality characteristics of morbidly obese patients admitted to hospital and intensive care units. J Crit Care 2011;26(2):180-185.

3. Fezeu L, Julia C, Henegar A, Bitu J, Hu FB, Grobbee DE, et al. Obesity is associated with higher risk of intensive care unit admission and death in influenza A (H1N1) patients: a systematic review and meta-analysis. Obes Rev 2011;12(8):653-659.

4. Malhotra A, Hillman D. Obesity and the lung: 3. Obesity, respiration and intensive care. Thorax 2008;63(10):925-931.

5. Miehsler W. Mortality, morbidity and special issues of obese ICU patients. Wien Med Wochenschr 2010;160(5):124-128.

6. Pedoto A. Lung physiology and obesity: anesthetic implications for thoracic procedures. Anesthesiol Res Pract 2012;2012:154208.

7. De Jong A, Molinari N, Sebbane M, Prades A, Futier E, Jung B, et al. Feasibility and effectiveness of prone position in morbidly obese patients with ARDS: a case-control clinical study. Chest 2013;143(6): 1554-1561.

8. Ciaglia P, Firsching R, Syniec C. Elective percutaneous dilatational tracheostomy: a new simple bedside procedure; preliminary report. Chest 1985;87(6):715-719.

9. Guinot PG, Zogheib E, Petiot S, Marienne JP, Guerin AM, Monet P, et al. Ultrasound-guided percutaneous tracheostomy in critically ill obese patients. Crit Care 2012;16(2):R40.

10. McCague A, Aljanabi H, Wong DT. Safety analysis of percutaneous dilational tracheostomies with bronchoscopy in the obese patient. Laryngoscope 2012;122(5):1031-1034.

11. Rosseland LA, Laake JH, Stubhaug A. Percutaneous dilatational tracheotomy in intensive care unit patients with increased bleeding risk or obesity. A prospective analysis of 1000 procedures. Acta Anaesthesiol Scand 2011;55(7):835-841.

12. Mansharamani NG, Koziel H, Garland R, LoCicero J 3rd, Critchlow J, Ernst A. Safety of bedside percutaneous dilatational tracheostomy in obese patients in the ICU. Chest 2000;117(5):1426-1429.

13. Pelosi P, Croci M, Ravagnan I, Vicardi P, Gattinoni L. Total respiratory system, lung, and chest wall mechanics in sedated-paralyzed postoperative morbidly obese patients. Chest 1996;109(1):144-151.

14. Martino JL, Stapleton RD, Wang M, Day AG, Cahill NE, Dixon AE, et al. Extreme obesity and outcomes in critically ill patients. Chest 2011;140(5):1198-1206.

15. Zittermann A, Becker T, Gummert JF, Börgermann J. Body mass index, cardiac surgery and clinical outcome: a single-center experience with 9125 patients. Nutr Metab Cardiovasc Dis 2014;24(2): 168-175.

16. ARDS Definition Task Force, Ranieri VM, Rubenfeld GD, Thompson BT, Ferguson ND, Caldwell E, et al. Acute respiratory distress syndrome: the Berlin Definition. JAMA 2012;307(23):2526-2533.

17. Morris AE, Stapleton RD, Rubenfeld GD, Hudson LD, Caldwell E, Steinberg KP. The association between body mass index and clinical outcomes in acute lung injury. Chest 2007;131(2):342-348.

18. O'Brien JM Jr, Phillips GS, Ali NA, Lucarelli M, Marsh CB, Lemeshow S. Body mass index is independently associated with hospital mortality in mechanically ventilated adults with acute lung injury. Crit Care Med 2006;34(3):738-744.

19. Hans GA, Lauwick S, Kaba A, Brichant JF, Joris JL. Postoperative respiratory problems in morbidly obese patients. Acta Anaesthesiol Belg 2009;60(3):169-175. 